\title{
Fricke, Michael / Langenhorst, Georg / Schlag, Thomas (Hg.) (2020). Jugendbibeln. Konzepte, Konkretionen, religionspädagogische Chancen. Freiburg: Herder. ISBN 978-3-45 I-38632-9. 262 Seiten.
}

\section{Egbert Ballhorn}

Technische Universität Dortmund (egbert.ballhorn@tu-dortmund.de)

Jugendbibeln sind ein besonderes Genre. Jugendliche stehen Erwachsenen deutlich näher als Kindern, und Kinderbibeln sind leichter zu definieren. Was macht eine Bibelausgabe zu einer Jugendbibel? Die Zielgruppenorientierung kann sich auf eine Textauswahl beziehen, die Entwicklungsthemen des Pubertätszeitalters aufnimmt, sie kann sich in der sprachlichen Gestaltung bzw. einer eigenen Form der Übersetzung niederschlagen, ebenso auch in bewussten Zusatz-, Rahmen- und Erläuterungstexten für den Kreis der Lesenden, oder auch im beigefügten Bildmaterial. Die Reflexion auf die mediale Form der Darbietung und ihre jeweilige kommunikative Eigenart steht noch eher am Anfang. So steht die Jugendbibel im Schnittfeld von Jugendkultur, Literaturtheorie, Entwicklungspsychologie, Bibeldidaktik und Exegese.

Der Tagungsband geht dem Thema in seinen unterschiedlichen Dimensionen nach. Einerseits geht es darum, dass Jugendbibeln Teilhabe nicht nur am Bibeltext, sondern am kollektiven Wissen und den Zugangsweisen der christlichen Gemeinschaft zur Bibel ermöglichen sollen, andererseits gehören diejenigen, die Jugendbibeln erstellen, den Jugendkulturen nicht selbst an, fehlt es ihnen also zumindest partiell an Teilhabe daran. Man muss mit einer „programmatischen Entzogenheit der Vorstellungswelten Jugendlicher rechnen" (244).

Der vorliegende Band geht der ganzen Bandbreite des Themas nach, dessen Wurzeln bereits in die frühe Neuzeit zurückreichen (Melchior). Die „Zubereitungsformen“ von Jugendbibeln beziehen sich auf den Gesamttext oder auf Auswahltexte. Dabei können Standardübersetzungen oder solche in Jugendsprache Verwendung finden bis hin zu Paraphrasen. Auch Kontextualisierungen durch Rahmentexte spielen eine Rolle, die sowohl Hintergrundinformationen und Deuteangebote als auch thematische Brücken in die Lebenswelten Jugendlicher anbieten wollen. Es kann mediale Erweiterungen des Textes geben, wobei es ganz klassische Illustrationen, aber auch Comics (Mangas, Graphic Novels) geben kann sowie crossmediale Ergänzungen und auch eigene Bibel-Apps. Die Summe der vielfältigen Beiträge ist: Bei Jugendlichen ist alles viel anspruchsvoller als bei Kindern. In der Pubertät kommt es zu Einbrüchen gegenüber dem Kinderglauben und damit auch gegenüber bisherigen Wahrnehmungsweisen der Bibel. Bibeltexte sind nicht mehr ohne weiteres anschlussfähig und müssen daher sehr durchdacht in Jugendwelten hinein und zu ihren Fragestellungen hin kontextualisiert werden. Die Gefahr, sich anzubiedern, besteht ständig. Daher kommt Langenhorst auch zu dem nüchternen Urteil: „Die ,Jugendbibel” wird sich niemals als eine eigenständige und vergleichbar erfolgreiche Gattung wie die ,Kinderbibel` etablieren" (69).

Im mittleren Teil des Bandes werden einzelne konkrete Bibelausgaben vorgestellt: die „Jugendbibel der katholischen Kirche“, die „Volxbibel“, die digitale Jugendbibel „KonApp“ sowie die Jugendbibel des BDKJ „WIR erzählen DIE BIBEL“. Hier kommen ausschließlich Autorinnen und Autoren dieser Bibeln zu Wort, die ihre eigenen Konzepte darlegen. Durch die Konkretionen werden die Wagnisse, aber auch Schwierigkeiten von Jugendbibeln gut deutlich, beispielsweise wenn in der "Volxbibel“ Jesus mit dem Moped nach Jerusalem einfährt oder das Wort vom „Licht der Welt“ so übersetzt wird: „Ihr seid wie ein Kühlschrank für diese Welt - ohne euch würde diese Welt vergammeln.“ Selbsterklärtes Ziel ist es, 
durch die Entscheidung für gewagte Auslegungsmöglichkeiten eine „auch noch in vielen Jahren verständliche und relevante Sprache“ (144) zu sprechen, die vor allem junge, entkirchlichte Menschen anspricht. Einen anderen Weg geht die Jugendbibel des BDKJ, die als Auswahlbibel die Texte durch fiktive Autorenkommentare einleitet und sie erstaunlicherweise nach der (vermuteten) Reihenfolge ihrer historischen Entstehungszeit ordnet. Hier wird der Versuch unternommen, einen historischen Zugang, der „kulturwissenschaftlich“ genannt wird, in erzählender Form zur normativen Brille der Textwahrnehmung zu machen.

Im letzten Teil des Buches werden Überblicksthemen geboten, so z. B. zum Thema „Jugend und Bibel in der Spätmoderne" von M. Steinkühler, die einen Ansatz von Lebenswelten und Lebensfragen her wählt und Narrationskompetenzen in den Mittelpunkt stellt. Wenn sie jedoch mythische und dogmatische Formen des Erzählens der Bibel zuordnet und spätmodernen Formen entgegensetzt, historisiert sie die Bibel auf eine Weise, die nicht nötig erscheint. Der von ihr vorgestellte für die Literatur der Spätmoderne typische „unzuverlässige Erzähler" (204) steht doch eigentlich in fruchtbarer Korrespondenz zum biblischen multiperspektivischen Erzählen mit seinen Leerstellen und Brüchen. Hier sind noch manche Gespräche zwischen Exegese und Religionspädagogik fruchtbar weiter zu führen. Anregend ist auch der Beitrag von H. Roose über das biblische Gespräch, das sehr stark subjektorientiert ist und auf Lektürekompetenzen und gemeinsamen Austausch setzt, was freilich erfordert, „im Unterricht einen ,unwahrscheinlichen Raum' zu schaffen, der bestimmten bewussten und unbewussten Regelhaftigkeiten des Unterrichts widerspricht" (189).

Instruktiv ist das Schlusskapitel (Schlag), das viele Aspekte von Jugendbibeln zusammenführt. Am Ende bleibt beim Rezensenten auch die Überlegung, ob neben die „Zubereitung“ von Bibeltexten für Jugendliche im Kontext von Jugendbibeln nicht noch stärker auch Reflexionen der Verwendungsorte und der Lektüreprozesse stehen sollten. Was ist der "Sitz im Leben“ von Jugendbibeln? Lesen überhaupt Jugendliche freiwillig Bibeltexte, oder tun sie es vor allem in sanft erzwungenen Kontexten? Im Gegensatz zu Kinderbibeln, in denen Textdarbietung und Illustrationen den Schwerpunkt ausmachen, nehmen Jugendbibeln noch viel stärker auf vielfältige Rezeptionsweisen und -welten der Jugendlichen Bezug. Da Jugendbibeln sehr stark gestaltete Ausgaben sind, üben sie auch eine stark lenkende Funktion aus. Insofern gehen Bibeltexte noch stärker in didaktische Situierungen hinüber, als es bei Kinderbibeln der Fall ist. Diesen Weg sollte man noch stärker beschreiten, weil viele Bibelausgaben sich bemühen, den Jugendlichen vieles zu erklären und darzubieten, statt Settings zu reflektieren und zu schaffen, in denen sie eigenständig sich zum Bibeltext verhalten können und Lesekompetenz entwickeln. Zu Jugendbibeln gehört vielleicht noch stärker das Nachdenken über Lese- und Rezeptionssituationen hinzu, was auch erforderlich macht, die Rolle der vermittelnden Personen deutlicher nicht nur in das Entwickeln, sondern in die Lesekonzepte der Bibelausgaben einzubinden. 